\title{
ANÁLISIS CUALITATIVO DE LA ATENCIÓN EN LOS SERVICIOS DE PLANIFICACIÓN FAMILIAR OFRECIDOS A PACIENTES QUECHUAHABLANTES EN AYACUCHO, PERÚ
}

\author{
Rebecca Irons $\mathbf{s}^{1, a}$
}

\author{
RESUMEN
}

Objetivos. El objetivo del estudio fue abordar y analizar la atención médica brindada a mujeres quechuas de bajos ingresos que usan el Seguro Integral de Salud (SIS) para acceder a los servicios de planificación familiar, y determinar si existe un temor significante hacia los métodos anticonceptivos por las esterilizaciones forzosas ocurridas en el pasado. Materiales y métodos. Se realizaron 100 entrevistas en profundidad en 70 mujeres, 10 esposos y 20 trabajadores de salud, en tres centros de salud de la provincia de Vilcashuamán en Ayacucho. Resultados. Se encontró que no existe un temor significativo hacia la planificación familiar por las esterilizaciones ocurridas en el pasado, tal como se planteó la hipótesis. Tampoco se halló una barrera lingüística significativa, ya que la mayoría de los trabajadores de salud hablaban quechua o estaban dispuestos a aprenderlo. Hubo algunos malentendidos y malos tratos entre pacientes y trabajadores de salud, referidos al uso de los servicios asistenciales que brinda el estado. Un temor generalizado sobre el cáncer fue evidente en relación con la anticoncepción. Conclusiones. No se encontró un temor significante hacia las esterilizaciones pasadas, por el contrario, las mujeres temen más a la relación entre el cáncer y la anticoncepción. La capacitación de los trabajadores de salud debe centrarse en comprender las perspectivas de los pacientes y reducir el estigma respecto a la pobreza, además de explicar las razones para ser beneficiario del SIS y del programa JUNTOS.

Palabras Claves: Planificación familiar; Salud reproductiva; Salud rural;Obstetricia; Anticoncepción (fuente: DeCS BIREME).

\section{QUALITATIVE ANALYSIS OF THE CARE IN THE FAMILY PLANNING SERVICES OFFERED QUECHUA-SPEAKING PATIENTS IN AYACUCHO, PERU}

\begin{abstract}
Objectives. The aim of this study was to address and analyze the medical care provided to low-income Quechua women who use the Seguro Integral de Salud (SIS, in Spanish) (comprehensive health insurance) to access family planning services, and to determine whether there is a significant fear toward contraceptive methods because of the forced sterilizations occurred in the past. Materials and Methods. One hundred (100) in-depth interviews were conducted with 70 women, 10 husbands, and 20 health workers in three health centers in the province of Vilcashuaman in Ayacucho. Results. It was found that there is no significant fear of family planning due to past sterilizations, as hypothesized. No significant language barrier was found either, as most health workers spoke or were willing to learn Quechua. There were some misunderstandings and abuse between patients and health workers referred to the use of healthcare services provided by the state. A widespread fear of contraception-related cancer became evident. Conclusions. No significant fear of past sterilizations was found; on the contrary, women are more afraid of the relationship between cancer and contraception. Health worker training should focus on understanding patient perspectives and reducing stigma around poverty, as well as explaining the reasons for being a recipient of the SIS and the "JUNTOS" program.
\end{abstract}

Keywords: Family Planning; Reproductive Health; Rural Health; Obstetrics; Contraception (source: MeSH NLM).

University College London, Reino Unido.

a Antropóloga, MRes

Este documento forma parte de la tesis doctoral «Planificación de familias quechuas: subjetividades, desigualdades y parentesco en el programa nacional de planificación familiar», para optar por el título de Doctor en Antropología Médica (PhD) de University College London, Reino Unido Recibido: 11/03/2019 Aprobado: 06/06/2019 En línea: 28/06/2019

Citar como: Irons R. Análisis cualitativo de la atención en los servicios de planificación familiar ofrecidos a pacientes quechuahablantes en Ayacucho, Perú. Rev Peru Med Exp Salud Pública. 2019;36(2):188-95. doi: http://dx.doi.org/10.17843/rpmesp.2019.362.4356. 


\section{INTRODUCCIÓN}

En el pasado, la atención médica en los servicios de planificaciónfamiliarhasidoobjetodeescrutinio, especialmente en las zonas rurales y empobrecidas del Perú. Durante 1996-2000, unos 300000 peruanos supuestamente fueron esterilizados durante la campaña de planificación familiar de la época ${ }^{(1-3)}$ y numerosos académicos responsabilizaron de estos abusos a los trabajadores de salud empleados por el Ministerio de Salud (MINSA) en ese momento, ofreciendo el programa Salud básica ${ }^{(4,5)}$. Se acusó a los empleados, no sólo de ofrecer servicios y tratamientos de calidad inferior ${ }^{(6)}$, sino de insultar y degradar a los pacientes, en particular a las mujeres rurales ${ }^{(1)}$. El programa de esterilizaciones masivas fue implementado a nivel nacional, y no solamente en zonas rurales con gente quechua o indígena. Esta historia reciente hace que los servicios de planificación familiar, que ofrece el MINSA, pudiera representar una preocupación actual.

Además, desde que se introdujo la política de parto intercultural en 2005, las mujeres quechuas reciben atención en salud materna dentro de los establecimientos del MINSA, y no a través de parteras como ocurría antes ${ }^{(7,8)}$. La mortalidad materna en Perú fue descrita como la segunda más alta de América Latina ${ }^{(8,9)}$, sin embargo, esta se ha reducido debido a intervenciones que fomentan el parto hospitalario, especialmente en zonas rurales.

No obstante, esto también significa que los trabajadores del MINSA tienen una mayor exposición e interacción con los problemas de salud reproductiva y materna de las pacientes, lo que brinda mejores oportunidades para la anticoncepción después del parto, y adecuados controles de embarazo durante la gestación. En esta mayor interacción, existe la oportunidad de abordar la necesidad no satisfecha de anticoncepción y brindar un mejor servicio a la población; sin embargo, también es necesario investigar la atención médica actual a fin de identificar problemas de coerción, discriminación o insultos, tal como presumiblemente ocurrió hace años por las esterilizaciones forzadas.

Por otro lado, Ayacucho ha sido el campo de desarrollo para algunos proyectos de salud sexual y reproductiva con el fin de empoderar a las mujeres, destacando REPROSALUD de USAID y Manuela Ramos. REPROSALUD fue implementado en Vilcashuamán, y aunque la Fundación Manuela Ramos sigue trabajando en el departamento, actualmente no desarrollan un proyecto de salud sexual y reproductiva en Vilcashuamán. La organización no gubernamental Chirapaq, que trabaja por los derechos de la gente indígena, opera en Vilcashuamán actualmente. Aunque no se enfoquen específicamente en la salud, Chirapaq empodera a la mujer indígena. Esto significa que las mujeres quechuas en esta área tienen un programa de apoyo que puede ayudarlas a resistir el maltrato, como lo ocurrido con las esterilizaciones forzadas.

\section{MENSAJES CLAVE}

Motivación para realizar el estudio. Existe una brecha entre la necesidad y el uso actual de los métodos anticonceptivos en las zonas rurales de Perú, particularlmente dentro de comunidades pobres.

Principales hallazgos. Se encontró que existe un temor generalizado hacia la relación entre el cáncer y los métodos anticonceptivos, además se evidencio malos tratos y malentendidos entre las pacientes quechas y los trabajadores de salud.

Implicancias. El estudio contribuye con sugerencias e información para mejorar los servicios de planificación familiar en zonas rurales del país.

Este documento utiliza una metodología cualitativa para abordar y analizar la atención médica brindada a mujeres quechuas de bajos ingresos que usan el SIS para acceder a los servicios de planificación familiar. A partir de esto, será posible abordar los problemas subyacentes que pueden resultar en la no utilización de servicios por parte del paciente, y será posible hacer sugerencias para mejorar la atención médica, basándose en estos hallazgos.

El objetivo del estudio fue abordar y analizar la atención médica brindada a mujeres quechuas de bajos ingresos que usan el Seguro Integral de Salud (SIS) para acceder a los servicios de planificación familiar, y averiguar si existe un temor significante hacia los métodos anticonceptivos por las esterilizaciones pasadas.

La hipótesis plantea que existiría un temor significativo a la planificación familiar basada en las esterilizaciones pasadas, y que existiría una barrera lingüística significativa entre los trabajadores de salud que sólo hablan castellano y los pacientes quechuahablantes.

\section{MATERIALES Y MÉTODOS}

La metodología de investigación utilizada fue cualitativa siguiendo la lista de verificación de 32 puntos de la guía COREQ ${ }^{(10)}$.

Todas las entrevistas fueron completadas por la autora del estudio, en castellano y en quechua básico. La investigadora posee una Maestría en Investigación (MRes), MA y BSc, y actualmente es candidata a doctorado en Antropología Médica en University College London (UCL). Ha completado estudios etnográficos con poblaciones rurales y urbanas en Perú y tiene una cualificación avanzada de capacitación en investigación. La investigadora y los participantes del estudio se conocieron el día de la entrevista en la mayoría de los casos o habían desarrollado una relación de trabajo durante un año. Los participantes fueron informados de los objetivos del estudio antes de 
consentir su participación. La institución de la investigadora (UCL) y la institución colaboradora (MINSA-Ayacucho) fueron informados de la realización del estudio.

El diseño del estudio utilizó una metodología cualitativa de investigación centrada en entrevistas en profundidad y observación dentro de una red de salud administrada por el MINSA en Vilcashuamán, Ayacucho. La investigación se basa en teorías antropológicas y sociológicas de la salud.

\section{SELECCIÓN DE PARTICIPANTES}

Los participantes fueron seleccionados utilizando muestreo por conveniencia en el personal de salud y muestreo en bola de nieve dentro de las comunidades seleccionadas. Todos los participantes fueron abordados cara a cara. Se entrevistó a un total de 100 participantes: 70 pacientes mujeres, 10 esposos y 20 empleados de salud. No se retiraron participantes. Uno de cada tres participantes rechazó que las entrevistas sean grabadas. Los datos se recolectaron dentro de los puestos de salud, centros de salud, hogares de los participantes y lugares de conveniencia (por ejemplo, su lugar de trabajo).

\section{RECOPILACIÓN DE LA INFORMACIÓN}

Todas las entrevistas se completaron sin intervención externa. La muestra tenía rangos de edad entre 18 y 49 años, disponían de SIS, se encontraban registrados en una red de salud en Ayacucho y hablaban quechua. La entrevista siguió un formato estructurado con indicaciones de la investigadora, para una explicación más detallada de las preguntas o para la discusión cuando sea apropiado. Las entrevistas fueron grabadas cuando se obtuvo el permiso del participante. Tuvieron una duración entre 15 a 45 minutos. No se repitieron entrevistas. Las entrevistas con participantes femeninas alcanzaron la saturación. Las transcripciones no fueron devueltas a los participantes.

\section{ANÁLISIS DE DATOS}

Los datos fueron codificados, no se utilizó un árbol de codificación. Los temas se derivaron una vez que todos los datos habían sido recopilados. El software de reconocimiento de voz Dragon versión 6.0.0 (www.nuance. com) se utilizó para ayudar con la transcripción de la entrevista. Los participantes se codificaron por edad, sexo y ciudad de residencia, pero no fueron numerados. Hubo coherencia entre los datos presentados y los hallazgos.

\section{CONSIDERACIÓN ÉTICAS}

El estudio fue sometido y aprobado por el comité ético de University College London y por la Dirección Regional de Salud de Ayacucho del Ministerio de Salud de Perú.
Tabla 1. Información sociodemográfica de los pacientes entrevistados.

\begin{tabular}{lcc}
\hline Características & $\mathbf{n = 8 0}$ & $\%$ \\
\hline Sexo & & \\
\hline Femenino & 70 & 87,5 \\
\hline Masculino & 10 & 12,5 \\
Grupo etario (años) & & \\
\hline $18-25$ & 27 & 33,8 \\
\hline $26-35$ & 24 & 30,0 \\
\hline $36-45$ & 17 & 21,2 \\
\hline >45 & 12 & 15,0 \\
\hline Profesión & & \\
\hline Ama de casa & 52 & 65,0 \\
\hline Agricultor & 22 & 27,5 \\
\hline Administración & 4 & 5,0 \\
\hline Otro & 2 & 2,5 \\
\hline
\end{tabular}

\section{RESULTADOS}

\section{CARACTERÍSTICAS DE LA POBLACIÓN ESTUDIADA.}

Todos los participantes hablaban quechua y castellano (aunque sus niveles en castellano variaron). La información sociodemográfica se detalla en la Tabla 1. El personal de salud estaba conformado por obstetras, enfermeras, médicos, técnicos, y trabajadores sociales. Debido a la poca cantidad de profesionales entrevistados, no se menciona el número, sexo ni edad de cada entrevistado, a fin de mantener la anonimidad de estos profesionales.

\section{ESTERILIZACIONES PASADAS}

En la provincia estudiada, se reportaron 153 esterilizaciones forzadas (Ministerio de Justicia y Derechos Humanos (MINJUSDH) ${ }^{(11)} .13$ de estas 153 personas se incluyeron en el estudio. A pesar de estos números, hubo poca o ninguna preocupación expresa porpartedel paciente sobrelaatención médica contemporánea en la planificación familiar debido a las esterilizaciones pasadas. Los pacientes entrevistados a veces expresaron dudas sobre si las esterilizaciones se hicieron cumplir o si fueron consensuadas:

«Ellos hubieran querido ser esterilizados, no puedes obligar a nadie a hacer esto» - (Hombre, 42)

«No nos pueden obligar hacer nada que no queramos hacer» (Mujer, 35).

\section{EL IDIOMA}

Todas las pacientes entrevistadas hablaban quechua como su idioma nativo, y todas eran competentes en castellano 
con diversos grados de fluidez. Todas las obstetras incluidas en el estudio pudieron comunicarse en quechua, además todas ellas habían estudiado en la Universidad Nacional San Cristóbal de Huamanga (UNSCH), donde recibieron clases de quechua como parte de su plan de estudios de capacitación obstétrica.

Hubo algunos trabajadores de salud que no hablaban quechua con fluidez, primordialmente aquellos que estaban realizando el Servicio Rural Urbano Marginal en Salud (SERUMS) y que no habían recibido capacitación en la UNSCH. Sin embargo, se observó que estos trabajadores realizaron estudios privado del idioma por iniciativa propia, compartiendo recursos entre ellos y practicando en su tiempo libre. Hubo un notable interés por interactuar con los pacientes en su idioma, o al menos, entender el quechua en un nivel básico.

Dentro de las áreas de espera y en las salas de consulta de los puestos de salud, había afiches que promovían la salud materna, tanto en castellano como en quechua (Figura 1). Se observó que los obstetras siempre comenzaban la consulta en castellano, y sólo cambiaban a quechua si el paciente no entendía el castellano.

\section{FRICCIÓN ENTRE PACIENTES Y TRABAJADORES DE $S A L U D$}

Entre los trabajadores de salud y las pacientes hubo mutuos sentimientos de malestar, ocasionado primordialmente por la atención gratuita recibida bajo la modalidad del SIS.

Los trabajadores de la salud percibían que las mujeres mostraban indiferencia por los servicios de planificación familiar y por la salud de sus hijos, ya que asumían que el estado pagaría por ellos sin importar lo que pasara.

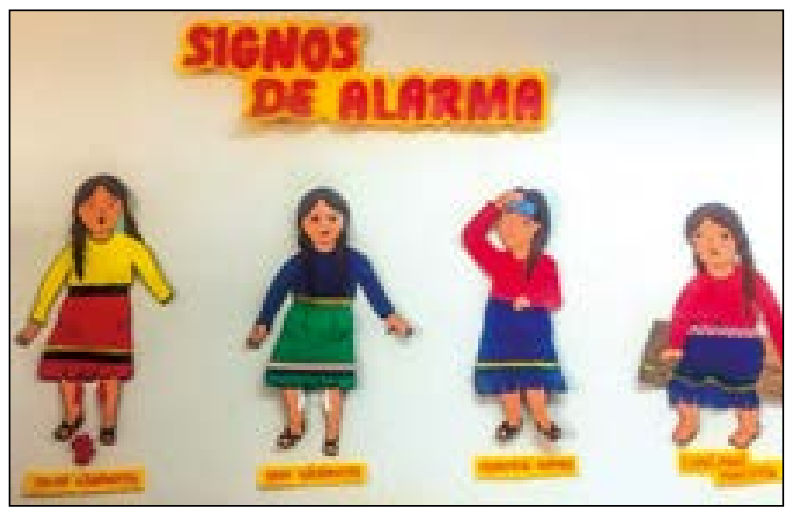

Figura 1. Signos de alarma en el caso del embarazo, ubicada en el consultorio de obstetricia en una posta de Ministerio de Salud, Ayacucho
Se observó a un obstetra comentando lo siguiente a una paciente posnatal:

"¿Quién va a pagar por todo esto? El estado paga por su embarazo, su vaso de leche, sus JUNTOS ... y si su bebé muere a causa de la anemia, ¿quién pagará? Es otra inversión más para el estado».

Otro trabajador de la salud argumenta que las mujeres deberían dejar de recibir subsidios del gobierno, especialmente aquellas que reciben dinero del programa JUNTOS:

«Si no les paga, ellos no harán nada, ni siquiera por sus hijos. Son bien interesadas. Estaba esta mujer de treinta y siete años que no acudiría a sus controles porque dijo que ya tenía cuatro hijos y sabía lo que estaba haciendo ... pero cómo no podía preocuparse por su bebé. Ella dijo, 'es mi cuerpo, yo tomo mis decisiones'. No deberían seguir dándoles dinero en JUNTOS».

Se observó a los obstetras sugiriendo a los pacientes que debían usar algunos de los métodos anticonceptivos (con mayor frecuencia, el implante de tres años), sin importancia que la mujer quería usar o no este método, lo que causó fricción entre ellos.

Por su parte, las pacientes estaban descontentas con este trato y no percibían que los trabajadores de salud tuvieran derecho a hablarles de esta manera. En su entrevista, una paciente comentó:

"¿Quiénes son para tratarnos así? Ellos sólo trabajan con la salud, ellos no pagan nada, yo pago mis cosas, que no se metan»(Mujer, 36).

\section{CÁNCER}

Las mujeres tenían la percepción que los métodos anticonceptivos hormonales, disponibles en los centros de salud, les podían provocar tumores o quistes cancerosos, y que los propios trabajadores de salud tenían la intención de contagiarlos con cáncer.

Las participantes creían que el contagio del cáncer ocurría durante los exámenes de Papanicolaou. Un obstetra comentó:

«Ellas dijeron que cuando hacemos Papanicolaou les contagiamos con cáncen».

Otro obstetra fue acusado de ser un «pishtaco» cuando se disponía a tomar una biopsia en pacientes femeninas. El "pishtaco» es considerado un personaje de la leyendaandina, que roba la sangre y la grasa de los habitantes andinos ${ }^{(17-19)}$. Anteriormente la comparación entre los trabajadores de salud y los «pishtacos» se había mencionado ${ }^{(20)}$, pero no específicamente en relación con los obstetras. 
Estas acusaciones estaban relacionadas con las campañas de Papanicolaou, donde muchas mujeres reciben la prueba en el mismo día que su consulta de planificación familiar. Un paciente afirmó que fue contagiada con un tumor durante uno de estos eventos:

«Hubo una campaña, muchas mujeres se habian ido, pero como habian ido muchas, no se cambiaron los guantes, y hasta ahora tengo una infección, por eso no puedo hacer nada. Tengo un tumorcito, yo también tengo una infección, yo estaba sanita, el día siguiente no mas me apareció, pregunté por qué, porque no se cambiaron los guantes para hacerlo rápido, porque había mucha gente, muchas mujeres, nos obligaron pe » (Mujer, 42).

Sin embargo, los temores sobre cáncer no sólo estaban relacionados con la atención que brindaban los trabajadores de salud, sino también por la percepción de acumulación de sangre que se produce por el uso de los métodos anticonceptivos hormonales. Las pacientes mostraron preocupación por la falta de sangrado menstrual cuando usaban los métodos anticonceptivos, específicamente la inyección de tres meses:

«A veces las mujeres, nos preocupamos cuando no hay sangre bajando, debes tener sangre todos los meses, dicen que se forma quiste, se forma cáncer señorita, dicen que forma un quiste, forma cáncer, se acumula adentro, se acumula en el interior, forma una bolita» (Mujer, 30)

"Con la ampolla, no había sangre, había dejado la ampolla y vino mucha sangre, ¿Por qué sería eso? ¿Acumulación de qué?» (Mujer, 27)

"Usé la ampolla, la sangre no vino durante muchos meses, y luego vino como coágulos, dicen que es cáncer, tumorcitos» (Mujer, 38)

Había una creencia generalizada de que la falta de sangre menstrual indicaba el crecimiento de un tumor o quiste dentro del cuello uterino, formado por la acumulación de sangre menstrual que no se había expulsado correctamente durante el período de uso de anticonceptivos.

A las pacientes mujeres se les solicita a menudo que se realicen la prueba de Papanicolaou cuando se presentaban para consulta de planificación familiar. Además, se puede observar en el centro de salud tiene un afiche que presenta al cáncer y a la planificación familiar juntos, surgiendo que hay una relación entre los dos (Figura 2).

\section{DISCUSIÓN}

Según Ballón ${ }^{(12)}$, el MINSA recomendó las esterilizaciones para mujeres que tenían más de tres hijos, estaban en riesgo obstétrico y tenían una edad mínima de 30 años. La edad promedio de las personas en la población del estudio fue de 50,4 años, lo que los ubicaría en alrededor de 30

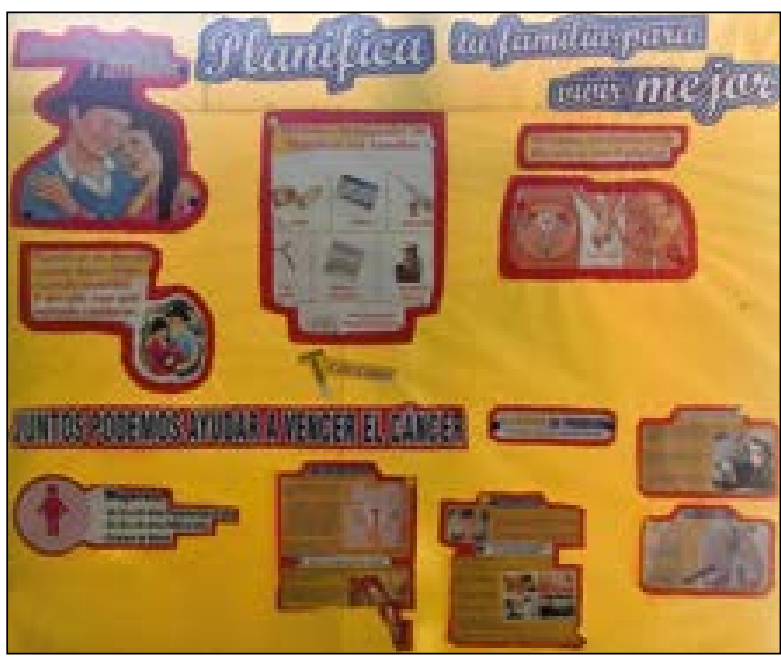

Figura 2. Afiche para promover la planificación familiar y la prueba Papanicolaou, ubicada en un consultorio obstétrica adentro de una posta de Ministerio de Salud, Ayacucho

años entre 1996-2000. El número promedio de niños fue de cuatro, cumpliendo con las supuestas regulaciones del MINSA. Sin embargo, las 13 víctimas reportadas tenían menos de tres hijos y un hombre no tenía hijos.

En la provincia estudiada también se reportaron esterilizaciones masculinas en el 3,87\% de los casos, esta cifra es menor que el promedio nacional de $7,48 \%{ }^{(12)}$. Todos eran quechua-hablantes y el 59\% eran habitantes de zonas rurales, sin embargo, esto puede reflejar la composición demográfica de la zona ya que la provincia tiene uno de los porcentajes más altos de quechuahablantes de todo el país con una población predominantemente rural $(68,3 \%){ }^{(13)}$. Por lo tanto, si bien la población que fue esterilizada era en gran parte de origen quechua y de procedencia rural, esto podría solo reflejar la demográfica general, por lo que al menos en este caso, estas características no necesariamente significan que la población quechua fue elegida específicamente.

Apesardel hecho de que se habían producidoesterilizaciones dentro de la región durante la campaña 1996-2000, en general las personas entrevistadas no informaron tener miedo a los servicios de planificación familiar basándose únicamente en este razonamiento. Puede haber una serie de motivos para esto. Para empezar, Rousseau ${ }^{(3,6)}$ y Ewig ${ }^{(1)}$ argumentan que la mayoría de los trabajadores de salud que realizaron las esterilizaciones provenían de Lima u otras regiones costeras del país, y por lo tanto eran desconocidos para las pacientes. Sin embargo, en la provincia estudiada, los obstetras eran familiares de las pacientes, y muchos habían crecido en la misma región. Las pacientes pudieron identificar a los trabajadores involucrados en la campaña de esterilización, por su nombre. Por lo tanto, pudieron asociar este evento con personas específicas y no con los puestos de salud o con el servicio de planificación familiar. 
Además, las cifras son relativamente bajas para la región, los que se han declarado forzosamente esterilizados representan sólo el $0,5 \%$ de la población de la provincia ${ }^{(11)}$. Por lo tanto, la baja cantidad de casos puede deberse a otros factores como la muerte o migración de la víctima, o simplemente su negativa a reportar la esterilización. La falta de preocupación sobre las esterilizaciones no indica que las personas no sientan temor a la planificación familiar, sino que este no fue un indicador clave de este problema.

Los trabajadores de salud mencionaron que las esterilizaciones eran una de las opciones más populares para las mujeres. Según el Instituto Nacional de Estadística e Informática (INEI) ${ }^{(13)}$, las esterilizaciones femeninas en Ayacucho aumentaron de $4,2 \%$ en $1996,5,3 \%$ en 2000 y $5,4 \%$ en 2009 .

Algunos investigadores ${ }^{(1,6,22)}$ han argumentado que las campañas anteriores de planificación familiar en áreas rurales no tuvieron éxito debido a que los trabajadores de salud no pudieron obtener el consentimiento del paciente por la falta de habilidad para comunicarse en su idioma, tal como presumiblemente ocurrió en el caso de esterilizaciones. En general, se sugiere que una barrera clave para la provisión de atención médica de calidad en las áreas rurales es el idioma ${ }^{(1,7,8)}$. Sin embargo, esto no sería aplicable a nuestro estudio, ya que todos los trabajadores hablaban quechua o intentaban aprenderlo. Esto puede deberse a que en la región sigue el sistema de Comunidades Locales de Administración en Salud (CLAS), donde un consejo consultivo local selecciona a los empleados para la contratación. Es posible que tengan candidatos preferidos que hablan el idioma local, lo que reduce la barrera del idioma entre trabajadores y pacientes.

También hubo intentos claros de incorporar el idioma en afiches de promoción de la salud dentro de las postas. Esto se aplica sólo a la salud materna y se habría implementado siguiendo las políticas de parto intercultural del $2005^{(7,8)}$. Sin embargo, no hubo trípticos en quechua sobre planificación familiar. El hecho de que los trabajadores primero hablaban español, aunque comprendan el quechua, también muestra una brecha en el intento de atender a los pacientes rurales de manera más efectiva. Este esfuerzo ha avanzado, pero estos ejemplos muestran que todavía hay un largo camino por recorrer.

La tensión evidenciada entre los trabajadores de salud y las pacientes parecen estar centrada en la dependencia económica de las mujeres hacia el estado, en lugar de atribuirlo al racismo, tal como lo han sugerido otros estudios (1). La ausencia de racismo puede deberse a que los trabajadores de salud provienen de la región. Sin embargo, hubo un claro desprecio por los pacientes que tienen familias numerosas y que dependen del gobierno para recibir apoyo financiero, a través de SIS, programa JUNTOS o similar. Bridges llama a estas actitudes como
«Welfare queens» ${ }^{(23)}$, es decir que las personas pueden estigmatizar y menospreciar a las mujeres más pobres, que no reciben un salario y que, en cambio, necesitan asistencia financiera estatal para criar a sus familias. Bridges argumenta que esto a menudo tiene un elemento de racismo, incluso cuando no es evidente. De cualquier manera, refleja una forma de violencia obstétrica hacia las mujeres que los trabajadores de la salud pueden percibir como inferiores por cualquier motivo, ya que, en base a este estigma pueden discriminar a los pacientes con respecto a los métodos anticonceptivos u otros servicios de salud reproductiva que ofrecen.

La Defensoría del Pueblo está actualmente preocupada por la tasa de violencia obstétrica en las comunidades andinas ${ }^{(24)}$, y la evidencia de este documento sugiere que esto puede presentarse especialmente cuando las mujeres son receptoras de múltiples formas de bienestar estatal tales como el SIS y el programa JUNTOS.

Casi la mitad de la población en Perú tiene una opinión desfavorable de la prueba de Papanicolaou (25), y las pacientes con menor nivel educativo son reacias a esta prueba ${ }^{(26)}$. En general, en Perú existe temor, estrés y vergüenza hacia la prueba Papanicolaou (14,15). La relación entre la percepción del cáncer y los métodos anticonceptivos ha sido descrita en un estudio antropológico en mujeres andinas ${ }^{(16)}$. Sin embargo, los resultados de este documento en realidad sugieren otras razones para el temor hacia el cáncer, especialmente la planificación familiar. En primer lugar, la planificación familiar y el cáncer se presentan a menudo juntos. Por ejemplo, las mujeres que tienen una consulta de planificación familiar se pueden realizar una prueba de Papanicolaou al mismo tiempo, pero no se les explica el fundamento. Como se mostró en el afiche estos dos conceptos se muestran juntos, sin la debida explicación en términos claros y no médicos para los pacientes. Por lo tanto, es lógico que las pacientes vinculen uno como causal del otro. Los temores sobre la acumulación de sangre y el cáncer son comunes en las comunidades quechuas (21), aunque esto no se ha relacionado directamente con la sospecha de que los trabajadores de la salud contagian a las pacientes con cáncer en los puestos de salud.

Además, la falta de comprensión de la forma en que funciona el cuerpo humano induce a las pacientes a creer que su sangre menstrual se ha acumulado, ya que no se les explica que la supresión de sangre menstrual ocurre durante el uso de la «ampolla» (método anticonceptivo). Por lo tanto, las pacientes parecen asumir que los métodos de planificación familiar les pueden provocar cáncer, y esta sería la razón por la que se les realiza la prueba de Papanicolaou en la consulta de planificación familiar.

Otros problemas surgen por la creencia de las pacientes de que el personal de salud trata de contagiarlas con cáncer, lo que genera desconfianza hacia estos servidores. Las mujeres quechuas pueden sentirse incómodas mostrando 
sus genitales ${ }^{(27)} y$, hacerlo en campaña grandes de tamizaje puede exacerbar el estrés, lo que conlleva a acusaciones contra los obstetras.

El estudio fue realizado por solo una investigadora, quien es una mujer europea, que domina el castellano, pero no habla quechua con fluidez. Para minimizar los sesgos del idioma, se realizó cada entrevista en castellano principalmente, y la transcripción fue realizada por la investigadora. Cuando un participante manifestó su deseo de hablar en quechua, la entrevista fue grabada con su permiso, y posteriormente analizada, con la posibilidad de consultar términos no entendibles con una profesora de quechua. Además, se debe reconocer que, quizás la identidad extranjera pudo causar desconfianza entre los participantes. Para sopesar este problema, la investigadora vivió un año en comunidades locales, por lo que la gente se acostumbró a ella y a su rol de investigadora dentro de la provincia.

Aunque la barrera del idioma no se presentó como un gran problema, se sugiere que, para mejorar la comunicación entre el personal y los pacientes, los nuevos miembros que no hablan quechua pudieran ser alentados a practicar con los otros que si dominan el idioma. Esto podría implicar la formación de clubes de aprendizaje de idiomas, en particular para los trabajadores del SERUMS que pueden no haber crecido en un entorno de habla quechua.

Los problemas que rodean la tensiones entre los pacientes y los trabajadores de salud sugieren la presencia de estigma hacia la gente económicamente empobrecida.
Esto es más difícil de abordar a nivel de los puestos de salud, sin embargo, el MINSA podría lo podría incorporar en la capacitación de sus trabajadores, y las universidades, como la UNSCH, podría incluirlo en sus cursos de educación médica, a fin de complementar la capacitación clínica.

Para superar los temores existentes, el vínculo entre la anticoncepción y el cáncer debe explicarse en forma clara y sencilla, en lugar de presentarse de forma ambigua. Es posible que muchos pacientes no entiendan los diagramas o los términos médicos usados. Se podrían impartir talleres en las comunidades locales, donde se discutan estos temores y se expliquen las percepciones erróneas como la acumulación de sangre menstrual y su relación con el cáncer.

La atención médica en los servicios de planificación familiar se puede mejorar con enfoques más concienzudos hacia las preocupaciones y entendimientos del paciente.

Agradecimientos: La autora quisiera agradecer a Lorena Roca de la Dirección Regional de Salud de Ayacucho del Ministerio de Salud de Perú por su ayuda con el proyecto. También quisiera agradecer todos los pacientes y trabajadores de salud que participaron en el estudio.

Contribuciones de autoría: RI hizo la investigación del campo, además analizó los datos y escribió el presente articulo.

Fuente de financiamiento: Wellcome Trust, Reino Unido. PhD Studentship in Medical Humanities, 2016-2020.

Conflicto de interés: Ninguno.

\section{REFERENCIAS BIBLIOGRÁFICAS}

1. Ewig C. Second-Wave Neoliberalism: Gender, Race and Health Sector Reform in Peru. Pennsylvania: University of Pennsylvania Press; 2010.

2. Tamayo León G. Nada personal. Reporte de Derechos Humanos sobre la Aplicación de Anticoncepción Quirúrgica en el Perú 1996 - 1998. Lima: Comité del América Latina y el Caribe para la Defensa de los Derechos de la Mujer (CLADEM); 1999. Disponible en: https://1996pnsrpf2000. files.wordpress.com/2011/07/cladem _ nada-personal.pdf

3. Rousseau S. Women's Citizenship in Peru. The Paradoxes of Neopopulism in Latin America. New York: Palgrave Macmillan; 2009.

4. Villegas MC. La Verdad de Una Mentira. El Caso de Trescientos mil Esterilizaciones Forzadas. Lima: Editorial Planeta; 2017.
5. Gianella Malca G. Los médicos peruanos y las esterilizaciones forzadas: la historia aún no termina. En: Ballón A, editor. Memorias del Caso peruano de Esterilización Forzada. Lima: Biblioteca Nacional del Perú; 2014.

6. Rousseau $S$. The politics of reproductive health in Peru: Gender and social policy in the global south. Social Politics. 2007;14(1):93-125. doi: 10.1093/sp/ jxm002.

7. Guerra Reyes L. Changing Birth in the Andes: Safe Motherhood, Culture and Policy in Peru. [Tesis Doctoral]. EEUU: University of Pittsburgh; 2013.

8. Physicians for Human Rights, Yamin AE, Cole J, Moore Simas TA, Brown M. Deadly Delays: Maternal Mortality in Peru. A Rights-Based Approach to Safe
Motherhood. Cambridge: Physicians for Human Rights; 2007.

9. Instituto Nacional de Estadística e Informática (INEI). Encuesta Demográfica y de Salud Familiar-ENDES 2014, Nacional y Departamental [Internet]. Lima: INEI; 2014. Disponible en: https:// www.dhsprogram.com/pubs/pdf/FR310/ FR310.pdf

10. Tong A. Sainsbury P, CraigJ. Consolidated criteria for reporting qualitative research (COREQ): a 32-item checklist for interviews and focus groups. Int J Qual Health Care. 2007;19(6):349-57. doi: 10.1093/intqhe/mzm042.

11. Ministerio de Justicia y Derechos Humanos (MINJUS). Reporte estadístico de casos de víctimas de esterilizaciones forzadas atendidos por el Centro Emergencia Mujer Vilcashuaman. Periodo 2017. Lima; MINJUS; 2017. 
12. Ballón A. Introducción. En: Ballon A, editor. Memorias del Caso peruano de Esterilización Forzada, Lima: Biblioteca Nacional del Perú; 2014.

13. Instituto Nacional de Estadística e Informática (INEI). Encuesta Permanente deEmpleo. INEI:Lima; 2017. Recuperado de: https://www.inei.gob.pe/bibliotecavirtual/boletines/informe-de-empleo/1/ $25 / 05 / 2019$

14. Winkler J. Coffey A, Handwerker W. Women's participation in a cervical cancer screening program in northern Peru. Health Educ Res. 2008;23(1):10-24. doi: 10/1093/her/cyll56.

15. Castro M, Morfin R, Sánchez SE; Roca J, Sánchez E, Williams MA. Nivel de Conocimiento sobre el cáncer cervical y el Papanicolaou en relación al temor, estrés o vergüenza al tamizaje: Estudio transversal en una comunidad pobre. Rev Per Ginecol Obstet. 2005;51(2):94-9.

16. Yon Leau C. Hablan las mujeres Andinas: preferencias reproductivas y anticoncepción. Lima: Movimiento Manuela Ramos; 2000.

17. Weismantel M. Cholas and Pishtacos: Stories of Race and Sex in the Andes. Chicago: University of Chicago press; 2001.
18. Wachtel N. Gods and Vampires: Return to Chipaya. Chicago: University of Chicago Press; 1994.

19. Oliver-Smith A. The Pishtaco: Institutionalized Fear in Highland Peru. J Am. Folklore. 1969; 82(32):363-8. doi: 10.2307/539781.

20. Bastien J. Drum and Stethoscope: Integrating Ethnomedicine and Biomedicine in Bolivia. Salt Lake City: University of Utah Press; 1992.

21. Hammer P. Bloodmakers Made of Blood: Quechua Ethnophysiology of Menstruation. En: Van de Walle E, Renne E, editors. Regulating Menstruation: Beliefs, Practices, Interpretations. London: University of Chicago Press; 2001.

22. Boesten J. Intersecting Inequalities: Women and Social Policy in Peru, 19902000. Pennsylvania: Pennsylvania State; 2010.

23. Bridges K. Reproducing Race: An Ethnography of Pregnancy as a Site of Racialization. Berkeley: University of California Press; 2011.

24. Defensoría del Pueblo. Derecho a la Salud Materna. Seguimiento de las recomendaciones defensoriales a establecimientos de salud en Arequipa, Junín, Lima, Piura y Puno. Lima:
Defensoría del Pueblo; 2016. [Citado el 25/05/2019]. Disponible en: https:// www.defensoria.gob.pe/modules/ Downloads/informes/varios/2017/ Informe-de-Adjuntia-N-001-2017-DPADM_Derecho-a-la-Salud-Materna-.pdf

25. Tenorio Galarza BJ, Tume Ruiz SRM. Percepciones y actitudes frente al examen de papanicolaou en mujeres atendidas en el Hospital Nacional Carrión [Tesis]. Lima: Universidad Mayor de San Marcos; 2004.

26. Huamani C, Hurtado-Ortega A, GuardiaRicra M, Mendoza J. Conocimientos y actitudes sobre la toma de Papanicolaou en mujeres de Lima, Perú 2007. Rev Peru Med Exp Salud Publica. 2008; 25(1):44-50.

27. Orr DM. Patterns of Persistence amidst medical pluralism: Pathways toward cure in the Southern Peruivan Andes. Med Anthropol. 2012;31(6):514-30. doi: $10.1080 / 01459740.2011 .636781$.

Correspondencia: Rebecca Irons

Dirección: Departamento de Antropologia, University College London, Reino Unido

Teléfono: +447727209342

Correo electrónico: rebecca.irons.14@ucl.ac.uk 